\title{
Trombose venosa cerebral e o uso de anticoncepcionais orais: Uma revisão integrativa
}

\author{
Cerebral venous thrombosis and the use oral contraceptives: An integrative review \\ Trombosis venosa cerebral y uso de anticonceptivos orales: Una revisión integradora
}

Recebido: 21/06/2021 | Revisado: 26/06/2021 | Aceito: 01/07/2021 | Publicado: 14/07/2021
Leonardo Soares Ribeiro Alves da Rocha
ORCID: https://orcid.org/0000-0002-1444-4839 Universidade do Oeste Paulista, Brasil
E-mail: leosrar12@gmail.com
Maria Eduarda Barres Vieira
ORCID: https://orcid.org/0000-0003-4793-4938 Universidade do Oeste Paulista, Brasil
E-mail: dudabarres@gmail.com
Angélica Augusta Grigoli Dominato
ORCID: https://orcid.org/0000-0001-6298-5106
Universidade do Oeste Paulista, Brasil
E-mail: angelica@unoeste.br

\begin{abstract}
Resumo
O objetivo do estudo foi relacionar as pesquisas sobre trombose venosa cerebral com o uso de anticoncepcionais orais combinados. Trata-se de uma revisão integrativa da literatura realizada através das bases de dados: LILACS, Scielo, Periódicos Capes, Medline PubMed, Cochrane Library e Science Direct, utilizando os descritores: "contraceptive agents", "intracranial embolism thrombosis", "thrombosis" "intracranial thrombosis". Para a seleção de artigos foi utilizado o operador booleano "AND" entre os descritores. A pergunta norteadora, elaborada através da metodologia de PICO (Paciente, Intervenção, Controle, Outcome), foi: "Os anticoncepcionais orais desencadeiam a trombose venosa cerebral?". Nos resultados foram encontrados 47 artigos, sendo 35 excluídos por não apresentarem relação entre trombose venosa cerebral e anticoncepcionais orais. Dessa forma, foram selecionados 12 artigos para compor essa revisão. No estudo pode-se concluir que, o risco dos eventos trombóticos venosos, em usuárias de anticoncepcionais orais combinados, está aumentado. Isto posto, é importante enfatizar o uso racional desses medicamentos, assim como a compreensão para o diagnóstico precoce da trombose venosa cerebral.
\end{abstract}

Palavras-chave: Anticoncepcionais orais; Trombose cerebral; Contracepção feminina.

\begin{abstract}
The aim of the study was to relate research on cerebral venous thrombosis with the use of combined oral contraceptives. This is an integrative literature review carried out using the following databases: LILACS, Scielo, Periódicos Capes, Medline PubMed, Cochrane Library and Science Direct, using the descriptors: "contraceptive agents", "intracranial embolism thrombosis", "thrombosis" "intracranial thrombosis". The Boolean operator "AND" was used between the descriptors to select the articles. The guiding question, elaborated through the PICO (Patient, Intervention, Control, Outcome) methodology, was: "Do oral contraceptives trigger cerebral venous thrombosis?". In the results, 47 articles were found, and 35 were excluded for not presenting a relationship between cerebral venous thrombosis and oral contraceptives. Thus, 12 articles were selected to compose this review. It can be concluded that the risk of venous thrombotic events in users of combined oral contraceptives is increased. Therefore, it is important to emphasize the rational use of these drugs, as well as the understanding for the early diagnosis of cerebral venous thrombosis.
\end{abstract}

Keywords: Oral contraceptives; Intracranial thrombosis; Female contraception.

\section{Resumen}

El objetivo del estudio fue relacionar los estudios sobre la trombosis venosa cerebral con el uso de anticonceptivos orales combinados. Se trata de una revisión integradora realizada a través de las siguientes bases de datos: LILACS, Scielo, Periódicos Capes, Medline PubMed, Cochrane Library y Science Direct, utilizando los descriptores: "contraceptive agents", "intracranial embolism thrombosis", "thrombosis" "intracranial thrombosis". Se utilizó el operador booleano "AND" entre los descriptores para seleccionar los artículos. La pregunta guía, desarrollada a través de la metodología PICO (Patient, Intervention, Control, Outcome), fue: "¿Los anticonceptivos orales desencadenan la trombosis venosa cerebral?". En los resultados se encontraron 47 artículos, siendo 35 excluidos por no presentar relación entre la trombosis venosa cerebral y los anticonceptivos orales. Así, se seleccionaron 12 artículos para componer esta revisión. El estudio permite concluir que el riesgo de eventos trombóticos venosos en usuarios de anticonceptivos orales 
combinados ha aumentado. Por lo tanto, es importante hacer hincapié en el uso racional de estos fármacos, así como en la comprensión para el diagnóstico temprano de la trombosis venosa cerebral.

Palabras clave: Anticonceptivos orales; Trombosis intracraneal; Anticoncepción para mujeres.

\section{Introdução}

Os anticoncepcionais orais (ACO) estão entre os métodos contraceptivos, compostos por hormônios femininos combinados, que desde a década de 1960, têm sido usados para o planejamento familiar (Luz et al., 2021). No Brasil, estima-se que aproximadamente $80 \%$ das mulheres na idade fértil fazem uso de algum tipo contracepção, incluindo o uso de ACO. Os métodos contraceptivos são classificados como reversíveis e definitivos, sendo os reversíveis identificados como: comportamentais, de barreira, hormonais, dispositivos intrauterinos e de emergência. Os definitivos ou irreversíveis são cirúrgicos (laqueadura das trompas de Falópio e a vasectomia) e tem observado diminuição drástica de sua aplicação (Silva et al., 2019; Miranda et al., 2016).

Ao longo do tempo os ACO têm apresentado evolução na formulação com a diminuição da dosagem dos hormônios, no intuito de minimizar o risco da ocorrência de hipertensão e trombose, além da evolução das morbidades causadas pelo uso ao longo da vida, especialmente quando associados ao tabagismo, hipertensão arterial, obesidade e dislipidemia (Luz et al., 202; Petitti, 2012).

Trombose é uma doença vascular cerebral causada pela oclusão dos seios venosos e/ou das veias cerebrais ocasionadas por trombos, que são inicialmente vermelhos, posteriormente substituídos por tecido fibroso e, que em alguns casos possui sinais de re-canalização (Souza et al., 2018). Dentre elas destaca-se a Trombose Cerebral, diferenciada entre Trombose Venosa Cerebral (TVC) e Trombose Arterial Cerebral (TAC). A TVC predomina em adultos jovens e, sobretudo em mulheres (cerca de 75\% dos casos no sexo feminino) (Callai et al., 2017). O tratamento precoce da TVC, ou seja no início do quadro clínico, exibe maior potencial de recuperação da paciente, quando comparada com as outras doenças cerebrovasculares (Souza et al., 2018).

O diagnóstico da TVC pode ser realizado através dos exames de imagem, como tomografia computadorizada de crânio (TCC) e ressonância magnética nuclear (RMN), aliado às manifestações clínicas na paciente, que podem ser diversificadas. Então, nos casos suspeitos, relacionados aos sintomas sugestivos, o tratamento precoce com anticoagulantes, inicialmente endovenoso e posteriormente oral, se faz necessário, mesmo que o resultado da TCC possa estar normal, em cerca de $20 \%$ a $25 \%$ das pacientes. Desta forma, existe um bom prognóstico para a maioria das pacientes (Santos et al., 2011; Renowden, 2004).

No entanto, é importante conhecer os riscos e/ou a predisposição à TVC, incluindo distúrbios do tipo prótrombóticos genéticos ou adquiridos, infecção do sistema venoso cerebral, neoplasias, doenças hematológicas, vasculites, doenças inflamatórias, sinusites, otites, gravidez, o puerpério, fatores existem também localmente como os tumores cerebrais, as mal formações arteriovenosas, traumas de crânio-encéfalo, quimioterapia e, especialmente, o uso de anticoncepcionais orais (Leal \& Bakker, 2017).

A TVC ocorre mais frequentemente em mulheres jovens, dos 20 aos 40 anos, estando diretamente ligados aos binômios homeostase hormonal e TVC. O uso de ACO, na contracepção ou reposição hormonal, estão presentes entre os grupos de maior frequência da TVC, atrelados a outras etiologias como distúrbios prótrombóticos. No entanto, um episódio de TVC em paciente fazendo o uso de ACO, sendo o único fator isolado de predisposição, aumenta a chance de evolução clínica favorável com o tratamento em relação àquelas com mais de um fator predisponente (Lima et al., 2017).

A TVC é uma doença, em geral, de boa evolução, que está diretamente relacionada com diagnóstico precoce e tratamento adequado. Porém, possui grande potencial para evoluir clinicamente desfavorável, com o óbito da paciente (Souza et al., 2018). Mesmo com o tratamento da TVC, a morbidade pode atingir os $20 \%$ e a mortalidade, geralmente está entre $5 \%$ a $10 \%$. Sendo assim, é necessário conhecer os possíveis fatores responsáveis pela TVC, bem como os aceitáveis prognósticos 
desfavoráveis que direcionam a terapêutica suplementar à anticoagulação mais adequada, considerando os benefícios e a segurança (Leal \& Bakker, 2017).

Dentre os fatores predisponentes para TVC, os hormonais estão mais relacionados especialmente quando associados ao uso de ACO. Então, o uso indiscriminado de ACO, por automedicação, uso prolongado e a presença de outros fatores de risco associados, aumentam o risco de TVC (Lima et al., 2017)

O objetivo foi relacionar os estudos sobre a Trombose Venosa Cerebral (TVC) e o uso de Anticoncepcionais Orais Combinados (ACO).

\section{Metodologia}

O estudo é uma revisão integrativa da literatura de natureza qualitativa e exploratória, que consistiu em buscas nas bases de dados (PubMed, Scielo, Lilacs, Science Direct, Cochrane) sobre o tema TVC associado ao uso de contraceptivos orais. A revisão integrativa permite ao profissional escolher os procedimentos ideais, no exercício diário de suas funções, através das evidências científicas, extraídas de estudos realizados anteriormente (Souza, Silva \& Carvalho, 2010). O estudo qualitativo e exploratório auxiliam na facilidade de interpretação do investigador sobre o tema estudado, aumentando a importância do entendimento sobre as variáveis em análise (Pereira, Shitsuka, Parreira \& Shitsuka, 2018; Köche, 2011).

A revisão integrativa utilizou o método PICO (Paciente, Intervenção, Controle, "Outcome" ou Resultado) para a formulação da pergunta, sendo Pacientes: mulheres que tiveram TVC fazendo o uso de contraceptivos orais combinados como fator de risco; Intervenção: contraceptivos orais combinados; Controle: fatores de risco para TVC; "Outcome", ou seja, resultado: uso de contraceptivos orais combinados e a TVC. Obteve-se então a pergunta norteadora para o estudo: "Os anticoncepcionais orais desencadeiam trombose venosa cerebral?"

O levantamento das referências foi realizado nas bases de dados: LILACS, Scielo, Periódicos Capes, Medline PubMed, Cochrane Library e Science Direct utilizando os seguintes descritores: "contraceptive agents", "intracranial embolism thrombosis", "thrombosis" "intracranial thrombosis" Para a seleção de artigos será utilizado o operador booleano "AND” entre os descritores.

Foram inclusos artigos originais que discorrem acerca da associação entre contraceptivos orais com a trombose e a trombose cerebral; nos idiomas inglês e português; artigos com o texto completo. Como critérios de exclusão: artigos repetidos; não responder a pergunta norteadora.

A busca dos artigos foi realizada por 2 autores. Primeiramente cada autor analisou os estudos encontrados nas bases de dados separadamente e após a coleta inicial, se reuniram para discutir os artigos filtrados e concluir a seleção dos mesmos. Os estudos incluídos eram ensaios clínicos randomizados, revisões integrativas, sistemáticas com meta-análises, experimental e estudo de coorte, publicados entre 2011 a 2020.

As informações extraídas dos estudos foram tempo de uso de contraceptivo, outros riscos de TVC associados à saúde da mulher, eficácia de cada método frente aos fatores de risco existentes para TVC, tratamentos, análise estatística e desfecho final.

Além disso, como parte da análise dos artigos recuperados, foi determinado o nível de evidência científica de cada um deles através do sistema de GRADE.

\section{Resultados}

As buscas realizadas nas plataformas de pesquisas resultaram em 47 artigos, sendo 35 excluídos por não apresentarem relação entre TVC e ACO. Ao final da seleção 12 artigos compuseram o estudo por atenderem aos critérios de inclusão. 
A análise e interpretação dos dados selecionados foram divididos nos quadros 1 e 2 contendo as principais informações extraídas dos artigos selecionados, como autor, ano, título, periódico, objetivo, tipo de estudo e principais resultados.

O Quadro 1 mostra que os anos de publicações dos estudos variaram de 2011 a 2020 em diferentes periódicos.

Quadro 1 - Autores, título, ano e periódico de publicação dos artigos utilizados no estudo.

\begin{tabular}{|c|c|c|c|}
\hline Autores & Título & Ano & Periódico \\
\hline Huang et al. & $\begin{array}{l}\text { A case report of oral contraceptive misuse induced } \\
\text { cerebral venous sinus thrombosis and dural } \\
\text { arteriovenous fistula. }\end{array}$ & 2019 & Medicine \\
\hline Panos et al. & $\begin{array}{l}\text { Unusual Presentation of Cerebral Venous Sinus } \\
\text { Thrombosis Associated with Contraceptive Usage. }\end{array}$ & 2014 & $\begin{array}{l}\text { Journal of Ophthalmic and } \\
\text { Vision Research }\end{array}$ \\
\hline Patil et al. & $\begin{array}{l}\text { Clinical profile and outcome of cerebral venous sinus } \\
\text { thrombosis at tertiary care center. }\end{array}$ & 2014 & J Neurosci Rural Pract \\
\hline Kesieme et al. & Deep vein thrombosis: a clinical review. & 2011 & Journal of Blood Medicine \\
\hline Bahal \& Santlal & $\begin{array}{l}\text { Norethisterone enanthate-induced cerebral venous } \\
\text { sinus thrombosis (CVST) }\end{array}$ & 2017 & BMJ \\
\hline Komro \& Findakly & $\begin{array}{l}\text { Cerebral Venous Sinus Thrombosis in Adults with } \\
\text { Prothrombotic Conditions: A Systematic Review and a } \\
\text { Case from Our Institution. }\end{array}$ & 2020 & Cureus \\
\hline Alvis-Miranda et al. & Cerebral sinus venous thrombosis. & 2013 & J Neurosci Rural Pract \\
\hline Gronich et al. & $\begin{array}{l}\text { Higher risk of venous thrombosis associated with } \\
\text { drospirenone-containing oral contraceptives: a } \\
\text { population-based cohort study. }\end{array}$ & 2011 & $\begin{array}{l}\text { Canadian Medical } \\
\text { Association or its licensors }\end{array}$ \\
\hline Xu et al. & $\begin{array}{l}\text { Oral contraceptives caused venous sinus thrombosis } \\
\text { complicated with cerebral artery infarction and } \\
\text { secondary epileptic seizures A case report and } \\
\text { literature review. }\end{array}$ & 2017 & Medicine \\
\hline Jick \& Hernandez & $\begin{array}{l}\text { Risk of non-fatal venous thromboembolism in women } \\
\text { using oral contraceptives containing drospirenone } \\
\text { compared with women using oral contraceptives } \\
\text { containing levonorgestrel: case-control study using } \\
\text { United States claims data. }\end{array}$ & 2011 & BMJ \\
\hline Stegeman et al. & $\begin{array}{l}\text { Different combined oral contraceptives and the risk of } \\
\text { venous thrombosis: systematic review and network } \\
\text { meta-analysis. }\end{array}$ & 2013 & BMJ \\
\hline Parkin et al. & $\begin{array}{l}\text { Risk of venous thromboembolism in users of oral } \\
\text { contraceptives containing drospirenone or } \\
\text { levonorgestrel: nested case-control study based on UK } \\
\text { General Practice Research Database. }\end{array}$ & 2011 & BMJ \\
\hline
\end{tabular}

Fonte: Autores (2021).

No Quadro 2 pode ser observado que os tipos de estudos foram variados como relato de caso, estudo observacional, retrospectivo e não intervencionista, revisões sistemática, da literatura, da bibliografia, caso controle e estudo de coorte. 
Quadro 2 - Distribuição dos estudos incluídos de acordo com autores, objetivo, tipo de estudo e principais resultados.

\begin{tabular}{|c|c|c|c|}
\hline Autor & Objetivo & Tipo de estudo & Principais resultados \\
\hline Huang et al. & $\begin{array}{l}\text { Apresentar relato de caso de } \\
\text { desenvolvimento crônico de } \\
\text { fístula arteriovenosa dural após } \\
\text { trombose do seio venoso cerebral } \\
\text { com uso de pílulas } \\
\text { anticoncepcionais orais para } \\
\text { aumentar a conscientização sobre } \\
\text { a doença no manejo clínico. }\end{array}$ & Relato de Caso. & $\begin{array}{l}\text { Uso de anticoncepcional oral: risco de trombose do seio } \\
\text { venoso cerebral. Pacientes com sintomas clínicos } \\
\text { inespecíficos (cefaléia e convulsões) suspeitar de } \\
\text { trombose do seio venoso cerebral. Exames de imagem } \\
\text { contribuíram para o diagnóstico evitando complicações } \\
\text { (dano permanente na parede do vaso, hemorragia cerebral } \\
\text { e fístula arterioventricular dural). }\end{array}$ \\
\hline Panos et al. & $\begin{array}{l}\text { Apresentar relato de caso de } \\
\text { trombose do seio venoso cerebral } \\
\text { associada ao uso de } \\
\text { anticoncepcional. }\end{array}$ & Relato de Caso. & $\begin{array}{l}\text { Presença de sintoma atípico (diplopia). As técnicas de } \\
\text { imagem são essenciais para o diagnóstico imediato e o } \\
\text { tratamento adequado de trombose do seio venoso } \\
\text { cerebral. }\end{array}$ \\
\hline Patil et al. & $\begin{array}{l}\text { Analisar as característcas da tvc } \\
\text { em um hospital rural. }\end{array}$ & $\begin{array}{l}\text { Estudo } \\
\text { retrospectivo, } \\
\text { observacional e } \\
\text { não } \\
\text { intervencionista. }\end{array}$ & $\begin{array}{l}\text { Sintomas comuns cefaleia e vômitos. Trombose do seio } \\
\text { sagital superior foi mais comum ao contrário da trombose } \\
\text { do seio cavernoso bilateral entre a trombose dos seios } \\
\text { venosos cerebrais. Um terço da população feminina foi } \\
\text { afetada no período periparto. A infecção e/ou } \\
\text { desidratação foi mais associado ao desenvolvimento de } \\
\text { trombose dos seios venosos centrais e mais de um quinto } \\
\text { da população apresentou evidência de hiper- } \\
\text { homocisteinemia. Mortalidade foi maior em pacientes } \\
\text { com trombose do seio sagital superior nas pacientes. }\end{array}$ \\
\hline Kesieme et al. & $\begin{array}{l}\text { Apresentar atualização sobre as } \\
\text { causas e o manejo da TVP. }\end{array}$ & $\begin{array}{l}\text { Revisão } \\
\text { bibliográfica. }\end{array}$ & $\begin{array}{l}\text { Profilaxia, mecânica e farmacológica (fondaparinux, um } \\
\text { inibidor seletivo indireto do fator Xa e os novos } \\
\text { inibidores orais seletivos diretos da trombina } \\
\text { (dabigatrana) e inibidores seletivos do fator Xa } \\
\text { (rivaroxabana e apixabana), da trombose venosa } \\
\text { profunda é importante. Tratamento de escolha com } \\
\text { anticoagulante, com heparina de baixo peso molecular, a } \\
\text { não fracionada e os antagonistas da vitamina K. }\end{array}$ \\
\hline Bahal \& Santlal & $\begin{array}{l}\text { Destacar a importância de } \\
\text { suspeita de trombose do seio } \\
\text { venoso cerebral em indivíduos } \\
\text { com progesterona de depósito de } \\
\text { longo prazo e tratamento } \\
\text { agressivo para resultados ótimos } \\
\text { e satisfatórios. }\end{array}$ & Relato de Caso & $\begin{array}{l}\text { Trombose do seio venoso cerebral condição rara que } \\
\text { pode mimetizar várias condições benignas, desta forma } \\
\text { suspeitar, identificando a etiologia e as condições } \\
\text { predisponentes, para facilitar um diagnóstico imediato. O } \\
\text { manejo da trombose do seio venoso cerebral deve ser } \\
\text { agressivo para garantir o melhor resultado, pois o } \\
\text { prognóstico geralmente é favorável. }\end{array}$ \\
\hline Komro \& Findakly & $\begin{array}{l}\text { Revisar as características do } \\
\text { paciente, fatores de risco, } \\
\text { características } \quad \text { clínicas, } \\
\text { modalidades de tratamento e } \\
\text { resultados de trombose do seio } \\
\text { venoso cerebral em pacientes } \\
\text { com estados pró-trombóticos. }\end{array}$ & $\begin{array}{l}\text { Revisão } \\
\text { Sistemática. }\end{array}$ & $\begin{array}{l}\text { Suspeitar de trombose venosa cerebral para diagnóstico } \\
\text { precoce nos casos de contexto clínico adequado, para o } \\
\text { uso de anticoagulação ideal e em longo prazo. }\end{array}$ \\
\hline Alvis-Miranda et al. & $\begin{array}{l}\text { Revisar o conhecimento sobre a } \\
\text { trombose de seio venoso } \\
\text { cerebral. }\end{array}$ & $\begin{array}{l}\text { Revisão } \\
\text { Bibliográfica. }\end{array}$ & $\begin{array}{l}\text { Trombose de seio venoso cerebral é condição } \\
\text { multifatorial com causas especificas de gênero, com as } \\
\text { principais causas sendo diferentes nos países } \\
\text { desenvolvidos e em desenvolvimento. }\end{array}$ \\
\hline
\end{tabular}




\begin{tabular}{|c|c|c|c|}
\hline Gronich et al & $\begin{array}{l}\text { Investigar o risco de eventos } \\
\text { tromboticos venosos e arteriais } \\
\text { entre usuárias de vários } \\
\text { anticoncepcionais orais. }\end{array}$ & $\begin{array}{l}\text { Estudo } \\
\text { Coorte. }\end{array}$ & $\begin{array}{l}\text { O uso crescente de anticoncepcionais contendo } \\
\text { drospirenona, aumenta o risco de TVC então é importante } \\
\text { conscientizar a paciente. Embora o risco de } \\
\text { tromboembolismo venoso seja pequeno em relação às } \\
\text { pílulas de terceira geração, informar a paciente sobre o } \\
\text { problema é primordial, especialmente entre as mais } \\
\text { velhas ou obesas. }\end{array}$ \\
\hline $\mathrm{Xu}$ et al. & $\begin{array}{l}\text { Relatar caso de trombse venosa } \\
\text { cerebral complicada com infarto } \\
\text { da artéria cerebral e convulsões } \\
\text { epilépticas secundárias devido a } \\
\text { anticoncepcionais orais. }\end{array}$ & $\begin{array}{l}\text { Relato de caso e } \\
\text { revisão de } \\
\text { literatura }\end{array}$ & $\begin{array}{l}\text { Diagnóstico precoce, avaliação correta e tratamento } \\
\text { padrão ainda são desafios importantes para a trombose } \\
\text { venosa cerebral. Tratamento ativo é recomendado. }\end{array}$ \\
\hline Jick \& Hernandez & $\begin{array}{l}\text { Comparar o risco de } \\
\text { tromboembolismo venoso não } \\
\text { fatal no uso de contraceptivos } \\
\text { orais contendo drospirenona com } \\
\text { o daqueles r contendo } \\
\text { levonorgestrel. }\end{array}$ & $\begin{array}{l}\text { Projeto de caso- } \\
\text { controle } \\
\text { aninhado e e } \\
\text { estudo de coorte. }\end{array}$ & $\begin{array}{l}\text { Risco de tromboembolismo venoso não fatal entre } \\
\text { usuárias de anticoncepcionais orais contendo } \\
\text { drospirenona parece ser cerca de duas vezes maior do que } \\
\text { entre usuárias de anticoncepcionais orais contendo } \\
\text { levonorgestrel, após os efeitos de potenciais fatores de } \\
\text { confusão e vieses de prescrição terem sido levados em } \\
\text { consideração. }\end{array}$ \\
\hline Stegeman et al. & $\begin{array}{l}\text { Fornecer uma visão abrangente } \\
\text { do risco de trombose venosa em } \\
\text { mulheres que usam diferentes } \\
\text { anticoncepcionais } \\
\text { combinados. }\end{array}$ & $\begin{array}{ll}\begin{array}{l}\text { Revisão } \\
\text { sistemática }\end{array} & \text { e } \\
\text { meta-análise } & \text { de } \\
\text { rede. } & \end{array}$ & $\begin{array}{l}\text { Todos os anticoncepcionais orais combinados } \\
\text { investigados nesta análise foram associados ao risco } \\
\text { aumentado de trombose venosa. O tamanho do efeito } \\
\text { depende do progestágeno usado e da dose de } \\
\text { etinilestradiol. }\end{array}$ \\
\hline Parkin et al. & $\begin{array}{l}\text { Examinar o risco de } \\
\text { tromboembolismo } r \text { venoso } \\
\text { idiopático não fatal em usuárias } \\
\text { de anticoncepcional oral } \\
\text { combinado r contendo } \\
\text { drospirenona, em relação às } \\
\text { preparações de levonorgestrel. }\end{array}$ & $\begin{array}{l}\text { Estudo de caso- } \\
\text { controle } \\
\text { aninhado. }\end{array}$ & $\begin{array}{l}\text { Esses achados contribuem para a evidência emergente de } \\
\text { que o anticoncepcional oral combinado contendo } \\
\text { drospirenona carrega um risco maior de } \\
\text { tromboembolismo venoso do que as formulações } \\
\text { contendo levonorgestrel. }\end{array}$ \\
\hline
\end{tabular}

Fonte: Autores (2021).

\section{Discussão}

A terapia hormonal oral é o método preferido de contracepção, entre as mulheres jovens. Gronich et al. (2011) mostraram que o uso de anticoncepcionais orais combinados contendo drospirenona foi associado ao risco aumentado de eventos trombóticos venosos, mas não eventos trombóticos arteriais. Os fatores de risco incluíram idade avançada, obesidade e histórico de câncer, além do risco ser maior nos primeiros quatro meses de uso. Relataram também que o uso de anticoncepcionais orais confere risco absoluto de trombose venosa de 6,29 por 10.000 mulheres-ano.

Segundo Huang et al. (2019) o consumo de pílulas anticoncepcionais orais é um fator de risco conhecido para trombose do seio venoso cerebral entre as mulheres. Panos et al. (2014) analisaram a incidência de tromboembolismo pulmonar em mulheres sem uso de ACO, sendo 1,64\% em pacientes com distúrbios trombolíticos e 0,18\% em pacientes normais, sabendo que o uso de Anticoncepcional Oral Combinado eleva em 3 vezes o risco de Tromboembolismo Venoso. Patil et al. (2014) descreveram que a trombose dos seios venosos cerebrais podem ser causadas por uma série de estados pró-trombóticos e distúrbios do sistema de coagulação, como a causa hereditária de resistência à proteína $\mathrm{C}$, secundária ao polimorfismo do fator $\mathrm{V}$ de Leiden, resistência à proteína $\mathrm{C}$ e $\mathrm{S}$ e deficiência da antitrombina III. Os sintomas mais comuns foram cefaléia, vômitos e convulsão. Enquanto que, Kesieme et al. (2011) definiram a trombose venosa profunda como a formação de coágulos sanguíneos 
em veias profundas. Desta forma, o tratamento farmacológico utiliza terapia anticoagulante, como o uso de antagonistas da vitamina $\mathrm{K}$, heparina não fracionada e a heparina de baixo peso molecular.

Kesieme et al. (2011) discutiram que os anticoncepcionais orais combinados estão associados ao risco de duas a seis vezes maior de trombose venosa. Além disso, constata que o composto de estrogênio (etinilestradiol) em pílulas anticoncepcionais pode causar o aumento do risco de trombose.

Kmro, Findakly (2020) caracterizam a trombose do seio venoso cerebral como uma condição rara, com pressão intracraniana prejudicando a drenagem venosa cerebral, podendo levar a consequências fatais.

De acordo com Alvis-Miranda et al. (2013) o prognóstico do paciente com trombose de seio venoso cerebral depende da detecção precoce. Os pacientes devem ser submetidos à venografia por tomografia computadorizada e a investigação da causa geradora. Existem alguns fatores de risco relacionados ao gênero feminino, sendo eles: uso de anticoncepcionais orais combinados, gravidez, puerpério e terapia de reposição hormonal.

Xu et al. (2017) relataram que o anticoncepcional oral é um fator de risco amplamente conhecido para trombose venosa cerebral, sendo como incidência de Trombose venosa cerebral, cerca de $0,5 \%$ de todos os acidentes vasculares cerebrais.

Jick, Hernandez (2011) mostram que o risco de tromboembolismo venoso está associado às doses de estrogênio. Contraceptivos orais contendo drospirenona possuem risco de duas vezes maior do que entre usuárias de contraceptivos orais contendo levonorgestrel.

Stegeman et al. (2013) relataram que todos os contraceptivos orais investigados foram associados a um risco aumentado de trombose venosa. O tamanho do efeito depende do tanto do progestágeno usado quanto da dose de etinilestradiol.

Parkin et al. (2011) mostraram que contraceptivos com drospiredona possui 3 vezes mais riscos de episódios tromboembolicos em relação ao uso de levonorgestrel.

\section{Conclusão}

O risco de eventos trombóticos venosos em usuárias de anticoncepcionais orais combinados estão aumentados. Desta forma é importante conscientizar as pacientes que fazem uso principalmente de estrogênio, sobre o tromboembolismo venoso, especialmente nas pacientes mais velhas, obesas ou que estão no puerpério.

Além disso, o uso do contraceptivo oral drospeirenona está associado a um aumento três vezes maior de tromboembolismo venoso idiopático não fatal quando comparado com as pílulas contendo levonorgestrel.

Assim, além da importância do uso racional do medicamento anticoncepcional oral combinado e do alerta quanto aos eventos tromboembólicos, faz-se também necessário que os médicos compreendam a relevância de um diagnóstico precoce com uma avaliação correta e um tratamento padrão quando a trombose venosa cerebral ocorre, porém, sabemos que esses fatos ainda são desafios na atualidade médica.

Desta forma, é importante que outros estudos sejam realizados abordando os efeitos dos ACO sobre a trombose venosa cerebral, no intuito de tornar a terapêutica mais segura para as mulheres sem predisposição aos eventos trombóticos.

\section{Referências}

Bahall, M., \& Santlal, M. (2017). Norethisterone enanthate-induced cerebral venous sinus thrombosis (CVST). BMJ Case Reports, 2017, bcr-2017-222418. https://doi.org/10.1136/bcr-2017-222418

Callai, T., Daronco, F., Konrad, N. L., Wichmann, J. F., Costa, F., \& Prezzi, S. H. (2017). Tabagismo e uso de anticoncepcionais orais relacionados a fenômenos tromboembólicos: Relato de caso e revisão de literatura. Reprodução \& Climatério, 32(2), 138-144. https://doi.org/10.1016/j.recli.2016.11.004

Gronich, N., Lavi, I., \& Rennert, G. (2011). Higher risk of venous thrombosis associated with drospirenone-containing oral contraceptives: A population-based cohort study. CMAJ: Canadian Medical Association Journal = Journal de l'Association Medicale Canadienne, 183(18), E1319-1325. https://doi.org/10.1503/cmaj.110463 
Huang, Q., Chai, X., Xiao, C., \& Cao, X. (2019). A case report of oral contraceptive misuse induced cerebral venous sinus thrombosis and dural arteriovenous fistula. Medicine, 98(33), e16440. https://doi.org/10.1097/MD.0000000000016440

Jick, S. S., \& Hernandez, R. K. (2011). Risk of non-fatal venous thromboembolism in women using oral contraceptives containing drospirenone compared with women using oral contraceptives containing levonorgestrel: Case-control study using United States claims data. BMJ (Clinical Research Ed.), 342 , d2151. https://doi.org/10.1136/bmj.d2151

Kesieme, E., Kesieme, C., Jebbin, N., Irekpita, E., \& Dongo, A. (2011). Deep vein thrombosis: A clinical review. Journal of Blood Medicine, 2, 59-69. https://doi.org/10.2147/JBM.S19009

Köche, J. C. (2011). Fundamentos de metodologia científica: teoria da ciência e iniciação à pesquisa (Edição digital). Vozes.

Komro, J., \& Findakly, D. ([s.d.]). Cerebral Venous Sinus Thrombosis in Adults with Prothrombotic Conditions: A Systematic Review and a Case from Our Institution. Cureus, 12(4), e7654. https://doi.org/10.7759/cureus.7654

Leal, T., \& Bakker, B. (2017). A mulher bioquímica: Invenções do feminino a partir de discursos sobre a pílula anticoncepcional. Revista Eletrônica de Comunicação, Informação e Inovação em Saúde, 11(3), Article 3. https://doi.org/10.29397/reciis.v11i3.1303

Lima, A. C. S., Martins, L. C. G., Lopes, M. V. de O., Araújo, T. L. de, Lima, F. E. T., Aquino, P. de S., \& Moura, E. R. F. (2017). Influence of hormonal contraceptives and the occurrence of stroke: Integrative review. Revista Brasileira de Enfermagem, 70, 647-655. https://doi.org/10.1590/0034-7167-2016-0056

Luz, A. L. R., Barros, L. de S. R., \& Branco, A. C. da S. C. (2021). Métodos contraceptivos: Principais riscos e efeitos adversos. Revista de Casos e Consultoria, 12(1), e24112-e24112.

Miranda, A. A. M., Silva, C. G. de O. e, Thimoteo, G. M., Assis, L. F., Del'Duca, A., Carvalho, A. R. de, \& Miranda, J. P. L. de. (2016). Conhecimentos acerca de dst/aids e métodos contraceptivos dos discentes dos cursos integrados do IF sudeste MG - campus Juiz de Fora, Brasil - Knowledge of STD/AIDS and Contraceptive Methods in Students of Integrated Techinical Courses at the IF Sudeste M. Multiverso: Revista Eletrônica do Campus Juiz de Fora - IF Sudeste $M G, 1(1), 25-36$

Panos, G. D., Panos, L. D., Digklia, A., Hafezi, F., \& Gatzioufas, Z. (2014). Unusual Presentation of Cerebral Venous Sinus Thrombosis Associated with Contraceptive Usage. Journal of Ophthalmic \& Vision Research, 9(2), 281-284.

Parkin, L., Sharples, K., Hernandez, R. K., \& Jick, S. S. (2011). Risk of venous thromboembolism in users of oral contraceptives containing drospirenone or levonorgestrel: Nested case-control study based on UK General Practice Research Database. BMJ (Clinical Research Ed.), 342, d2139. https://doi.org/10.1136/bmj.d2139

Patil, V. C., Choraria, K., Desai, N., \& Agrawal, S. (2014). Clinical profile and outcome of cerebral venous sinus thrombosis at tertiary care center. Journal of Neurosciences in Rural Practice, 5(3), 218-224. https://doi.org/10.4103/0976-3147.133559

Pereira, A. S., Shitsuka, D. M., Parreira, F. J., \& Shitsuka, R. (2018). Metodologia da pesquisa científica. Núcleo de Tecnologia Educacional. UFSM.

Petitti, D. B. (2012). Hormonal Contraceptives and Arterial Thrombosis-Not Risk-free but Safe Enough. New England Journal of Medicine, 366(24), 23162318. https://doi.org/10.1056/NEJMe1204769

Renowden, S. (2004). Cerebral venous sinus thrombosis. European Radiology, 14(2), 215-226. https://doi.org/10.1007/s00330-003-2021-6

Santos, G. R., André, R., Pereira, S. L., Parreira, T., \& Machado, E. (2011). Trombose Venosa Cerebral: Análise Retrospectiva de 49 Casos. https://rihuc.huc.minsaude.pt/handle/10400.4/1018

Silva, C. S. da, Sá, R., \& Toledo, J. (2019). Métodos Contraceptivos e Prevalência de Mulheres Adultas e Jovens com risco de Trombose, no Campus Centro Universitário do Distrito Federal-UDF. REVISA (Online), 190-197.

Souza, N., Holanda, C., Holanda, V., Zapata, M., de Oliveira, J., \& Ribeiro, C. (2018). Trombose venosa de múltiplos seios: Relato de caso. 37. https://doi.org/10.1055/s-0038-1672736

Souza, M. T., Silva, M. D. \& Carvalho, R. (2010). Revisão Integrativa: o que é e como fazer Einstein. 8(1), 102-106. https://doi.org/10.1590/S167945082010RW1134

Stegeman, B. H., de Bastos, M., Rosendaal, F. R., van Hylckama Vlieg, A., Helmerhorst, F. M., Stijnen, T., \& Dekkers, O. M. (2013). Different combined oral contraceptives and the risk of venous thrombosis: Systematic review and network meta-analysis. BMJ (Clinical Research Ed.), 347, f5298. https://doi.org/10.1136/bmj.f5298

Xu, F., Liu, C., \& Huang, X. (2017). Oral contraceptives caused venous sinus thrombosis complicated with cerebral artery infarction and secondary epileptic seizures: A case report and literature review. Medicine, 96(51), e9383. https://doi.org/10.1097/MD.0000000000009383 